\title{
TREATMENT OF DERMATOPHYTE ONYCHOMY- COSIS WITH TERBINAFINE (LAMISIL) PULSE THERAPY
}

\author{
Sonia Valkova \\ Department of Dermatology and Venereology, \\ University Hospital - Pleven
}

\begin{abstract}
Terbinafine is the most active currently available antidermatophyte agent with fungicidal action. The aim of the present study was to assess the efficacy of terbinafine pulse therapy in the treatment of dermatophyte nail infections. Seven patients (two with fingernail, four with toenail and one with mixed onychomycosis) have been treated with $500 \mathrm{mg}$ terbinafine daily for one week per month. The duration of the treatment was four months for toenail and three months for fingernail onychomycosis. At the end of the follow-up period (six months after discontinuation of treatment) 6 out of 7 patients (87\%) were mycologically cured. In conclusion, pulse terbinafine therapy is an effective regimen for the treatment of dermatophyte nail infections.
\end{abstract}

Key words: onychomycosis, terbinafine, pulse therapy

\section{INTRODUCTION}

Terbinafine is an allylamine derivative with fungicidal properties against dermatophytes. It reaches the distal nail one week after initiation of therapy and persists in the nail plate for 2 to 3 months after treatment is stopped (3). Terbinafine at a dosage of $250 \mathrm{mg}$ daily produces cure rates of more than $80 \%$ in fingernail and toenail infections in 6 and 12 weeks, respectively.

At present pulse therapy with itraconazole $(400 \mathrm{mg}$ daily) is also recommended in the treatment of onychomycosis with cure rates ranging from $64 \%$ to $72 \%$ (2).

\section{AIM}

The aim of the present study was to verify whether pulse therapy with terbinafine is effective in the treatment of dermatophyte nail infections.

\section{PATIENTS AND METHODS}

Seven patients (4 men and 3 women) aged 33-65 years have been treated - four with toenail, two with fingernail and 1 with mixed onychomycosis. The duration of the disease varied from 1 to 8 years. Diagnosis was based on clinical data and mycological examination (demonstra- tion of fungus in $\mathrm{KOH}$ preparation in all patients and culture on Sabouraud's medium in four of them). Trichophyton rubrum was isolated in 3 of the patients and Trichophyton soudanense in one. The nail plate degree of involvement varied from $75 \%$ to $100 \%$. Patients who had received systemic antifungal therapy eight weeks before treatment and those with severe liver, renal or cardio-vascular diseases were not included in the study.

Patients were given terbinafine (Lamisil, Novartis), two tablets daily $(500 \mathrm{mg}$ ) for one week every month. Duration of treatment was four months for toenail and three months for fingernail infections. All patients were examined monthly and 6 months after discontinuation of therapy. Mycologic evaluation was performed at the beginning and at the end of the therapeutic course and at the end of the follow-up period. Depending on clinical and laboratory data at the end of the sixth month patients were assigned to one of the following categories - not mycologically cured, mycologically cured with residual malformation and mycologically cured without residual malformation.

\section{RESULTS}

\section{Mycological examination}

Mycological examination after four months of therapy was negative in three (43\%) of the patients with toenail onychomycosis. At the end of the follow-up period results were negative in four $(57 \%)$ of the same patients. All cases with only fingernail onychomycosis were mycologically cured at the end of the three-month treatment period with terbinafine.

\section{Final assessment of therapeutic results}

Final assessment of patients at the end of the followup period demonstrated mycological cure without residual malformation in three patients with toenail onychomycosis. One ended therapy mycologically cured with residual malformation (small band of onycholysis and residual distal subungual hyperkeratosis) and one was not mycologically cured. All cases with fingernail onychomycosis only were mycologycally cured without residual malformation. 


\section{Tolerability}

Drug-related adverse effects were reported from one patient only - moderately strong itch. It was successfully infuenced by antihistamines and did not necessitate interruption of therapy.

\section{DISCUSSION}

Terbinafine (Lamisil) is an allylamine that inhibits the enzyme squalene-epoxidase, thus blocking the conversion of squalene to squalene epoxide in the biosynthesis pathway of ergosterol, an integral component of the fungal cell wall. Its action results in both a depletion of ergosterol, which has a fungistatic effect, together with an accumulation of squalene, which appears to be directly fungicidal. The minimum inhibitory concentration of terbinafine is very low and is equivalent to the minimal fungicidal concentration, demonstrating that this drug is really fungicidal in vitro.

Literature data about the excellent therapeutic efficacy of terbinafine in onychomycosis treatment were confirmed by the results of the present study. In addition the drug was well -tolerated and no serious side effects were registered.

Although terbinafine is demonstrably the most effec- tive agent in dermatophyte onycho-mycosis, a constant failure rate of $20-30 \%$ is found in all studies. In accordance with this in our group one patient $(14,3 \%)$ ended therapy not mycologically cured. In this case the infection persisted along narrow linear streaks on the toes. Tosti et al. (8) reported similar results after terbinafine treatment. Baran (unpublished data) believes that this occurs because the nail plate does not adhere to the nail bed there. Because terbinafine diffuses to the nail plate through the nail bed it might not reach therapeutic concentrations in such sites.

Tosti et al. (8) report intermittent ( $250 \mathrm{mg}$ daily) and pulse (500 mg daily) therapy with terbinafine are equally effective in the treatment of dermatophyte onychomycosis (cure rates of $94 \%$ and $80 \%$ respectively). Other authors find pulse therapy less effective ( $49 \%$ cure rate) (4). We achieved $71 \%$ cure rate. The differences in the therapeutic results are probably due to variables in the criteria for clinical and mycological cure or in the duration of the follow-up period. Nevertheless the superiority of terbinafine versus itraconazole has recently been supported by a systematic review of oral treatments for toenail onychomycosis (1) and nowadays terbinafine is considered the most powerful antidermatophyte agent active in vitro and in vivo $(6,7)$.

\section{REFERENCES:}

1. Crawford F, Young P, Godfrey C et al. Oral treatments for toenail onychomycosis. A systematic review. Arch Dermatol 2002;138:811-816.

2. De Doncker P, Decroix J, Pierard G et al. Antifungal pulse therapy for onychomycosis. A pharmacokinetic and pharmacodynamic investigation of monthly cycles of 1-week pulse therapy with itraconazole. Arch Dermatol 1996;132(1):34-41.

3. Faegermann J, Zehender H, Millerioux L. Levels of terbinafine in plasma, stratum corneum, dermis-epidermis (without stratum corneum), sebum, hair and nails during and after $250 \mathrm{mg}$ terbinafine orally once daily for 7 and 14 days. Clin Exp Dermatol 1994; 19:121-126.

4. Gupta AK, Lynde CW, Konnikov N. Single-blind, randomized, prospective study of sequential itraconazole and terbinafine pulse compared with terbinafine pulse for the treatment of toenail onychomycosis. J Am Acad Dermatol 2001;44:485-491.

5. Munro CS, Shuster S. The route of rapid access of drugs to the distal nail plate. Acta Derm Venereol (Stockh) 1992; 72:387-388.

6. Ryder NS, Favre B. Antifungal ac- tivity and mechanism of action of terbinafine. Rev Contemp Pharmacother 1997; 8:275-288.

7. Ryder NS, Leither I. In vitro activity of terbinafine; an update. J Dermatol Treat 1998; 9( Suppl 1):S23-28.

8. Tosti A, Piraccini BM, Stinchi C et al. Treatment of dermatophyte nail infections: an open randomized study comparing intermittent terbinafine therapy with continuous terbinafine treatment and intermittent itraconazole therapy. J Am Acad Dermatol 1996; 34:595-600.

\author{
Address of the coresponding author: \\ Sonia Valkova \\ Clinic of Dermatology, \\ 91, Gen. Vladimir Vazov str., 5800 Pleven, Bulgaria \\ E-mail: soniderm@yahoo.com
}

